\title{
A statistical approach for measuring dislocations in 2D photonic crystals
}

\author{
Malureanu, Radu; Frandsen, Lars Hagedorn
}

Published in:

International Conference on Nanoscience and Nanotechnology

Link to article, DOI:

10.1109/ICONN.2008.4639281

Publication date:

2008

Document Version

Publisher's PDF, also known as Version of record

Link back to DTU Orbit

Citation (APA):

Malureanu, R., \& Frandsen, L. H. (2008). A statistical approach for measuring dislocations in 2D photonic crystals. In International Conference on Nanoscience and Nanotechnology (pp. 200-202). IEEE. https://doi.org/10.1109/ICONN.2008.4639281

\section{General rights}

Copyright and moral rights for the publications made accessible in the public portal are retained by the authors and/or other copyright owners and it is a condition of accessing publications that users recognise and abide by the legal requirements associated with these rights.

- Users may download and print one copy of any publication from the public portal for the purpose of private study or research.

- You may not further distribute the material or use it for any profit-making activity or commercial gain

- You may freely distribute the URL identifying the publication in the public portal

If you believe that this document breaches copyright please contact us providing details, and we will remove access to the work immediately and investigate your claim 


\title{
A Statistical Approach for Measuring Dislocations In 2D Photonic Crystals
}

\author{
$\underline{\text { R. Malureanu }}^{1}$, L.H. Frandsen ${ }^{1}$ \\ ${ }^{1}$ Dept. of Communications, Optics \& Materials, Technical University of Denmark, \\ Oersted Plads, 345v, 2800, Kgs. Lyngby, Denmark
}

\begin{abstract}
In this paper, a comparison between the placement accuracy of lattice atoms in photonic crystal structures fabricated with different lithographic techniques is made. Using atomic force microscopy measurements and self-developed algorithms for calculating the holes position within less than $0.01 \mathrm{~nm}$ error, we establish the statistical disorder within such devices.
\end{abstract}

Keywords - AFM measures, photonic crystals, placement accuracy

\section{INTRODUCTION}

The effect of various fabrication errors for the photonic crystals $(\mathrm{PhC})$ functionality is a very important issue that preoccupied the scientific community since the early stage of this field $[1,2]$.

Within this approach, the disorder in the holes' placement within a photonic crystal structure has been investigated both from the theoretical [3] and experimental [4] point of view in order to establish its influence over the structure's functionality. Due to lattice disorders on the order of nanometres the losses in such devices can increase dramatically thus degrading its performances.

In order to investigate the accuracy in placing low-index structures (air holes) in a high-index material using nowadays techniques, we collected samples made with different state-ofthe-art fabrication tools from various European research centres and, using the same measuring algorithm, mapped the lattice grid of each $\mathrm{PhC}$ structure and its error with respect to the ideal lattice parameters. All PhCs were defined by a triangular arrangement of air holes with diameter $\mathrm{D} \approx 200 \mathrm{~nm}$ and lattice constant $\Lambda=365 \mathrm{~nm}$.

\section{THE MEASURING SETUP}

For achieving high accuracy in measuring the topological map of the samples, we used an atomic force microscope (AFM). The setup is installed in stable room conditions that are essential for the quality of the measures and for obtaining high resolution. With the actual setup the direct-measure resolution is less than $1 \mathrm{~nm}$ allowing us to obtain, after averaging out, less than $0.01 \mathrm{~nm}$ resolution of the final parameters. To achieve such high resolution the AFM was operated in tapping mode that, apart from having higher resolution over other non-contact or contact possibilities, also has the advantage of having the lowest mechanical influence on the sample so sensitive structures (like membrane $\mathrm{PhCs}$ ) can be measured without loosing accuracy, i.e. without deforming the structure. After aligning the sample to the AFM tip and prior to measurements the AFM is left for several hours for thermal stabilisation. This is done, as the difference in temperature between the sample and the AFM holder will generate a thermal drift visible in the measures. Hence, a couple of hours of thermal stabilisation are necessary before the measures can be started.

As previously stated, the direct-measure error is in the $\mathrm{nm}$ range. One technique for minimising measuring errors is to average several measures and thus, obtain a better and more precise measure of the same area of the sample. Usually, at least 20 measures for each sample were taken, thus making a statistical approach possible. Also, for each measure both the forward and backward scan is recorded, thus giving 40 separate measures per structure that can be processed.

\section{POST-PROCESSING THE COLLECTED DATA}

The first step in post-processing the recorded data consists in applying a correction scheme to the mechanical errors mainly introduced by the mechanically non-perfect (on a micrometer scale) Cartesian coordinate system of the AFM. In order to obtain the correction parameters, a calibrated pattern is measured and then, by geometrical transformations, the obtained measure is matched with the real pattern's characteristics. The calibration parameters are defined by the mechanical tolerances of the AFM setup and can be applied for all measures, if one keeps the environment stable throughout the measurements. Our measures took place in a controlled (in terms of temperature and humidity) atmosphere so once determined the correction parameters are subsequently applied to all the measures.

The second step in the post-processing consists in levelling the structure horizontally (XY-plane). This is needed since the sample's plane and the measuring plane are not necessarily parallel. Moreover, planarization of the measure is needed, as the surface of the sample may have deformed while handling and mounting the sample. Such a deformation, even if it is a second-order approximation, can and does influences the measure. A final correction is the so-called "line-wise correction" that is applied in order to correct for the charging of the AFM tip that take place while measuring thus influencing the resonant frequency of the tip and the measured data. This type of error is critical since it influences the determination of the holes' border and thus the determination of their centre. 
To establish the position of each hole's centre we utilize a self-developed Matlab ${ }^{\mathrm{TM}}$ routine based on a centre-of-mass calculation. After the calibration steps described above, each measure is imported into Matlab ${ }^{\mathrm{TM}}$ and a recognising algorithm finds all the holes position and their centre of mass. In this way, we have for each measure a list of $(\mathrm{x}, \mathrm{y})$ pairs indicating the holes' position that can be used for further processing.

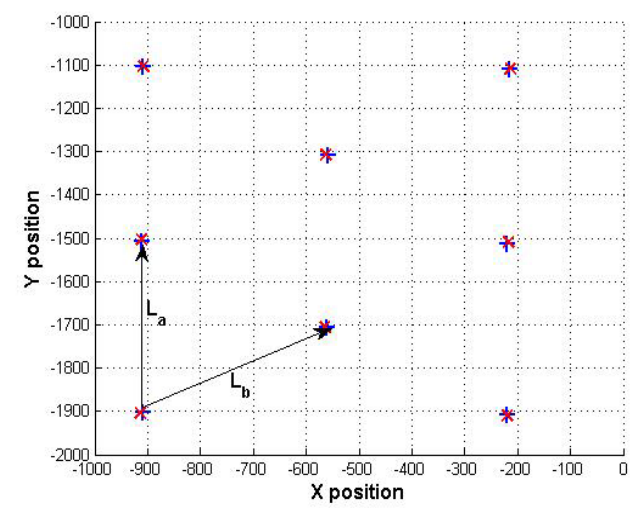

Figure 1. Lattice matching vectors and positions. The red " $x$ " represent the ideal matrix while the blue "+" the measured one

Due to the imperfect clamping of the sample to the sample holder, there is always a lateral drift in the measures. By averaging each forward scan with its corresponding backward scan this drift can be, if not eliminated at least greatly reduced. Due to the continuous measuring approach, for each forward measure there are two corresponding backward ones and viceversa so the total number of measures does not halves but diminishes with only one.

Once the averaged holes' positions are found, we use them as input for a least-square approximation method in order to determine the lattice that best matches the measured points (figure 1). The routine used for determining the lattice returns the 6 parameters that completely describe the lattice: the amplitude and angle of the two lattice vectors and the initial point of such a matrix.

Since the chamber conditions can vary during the measure (e.g. because of the outside temperature variation) and the thermal cycle feedback has a certain delay due to implicit inertia until re-establishing the initial conditions, thermal drift can appear even with the initial thermal stabilisation step. In order to track and eliminate such drift-influenced measures, we first plot the obtained lattice vectors' amplitudes so that a direct correlation between the two can be established (see figure 2). In the case of a thermal drift, due to the different orientation of the vectors, the change between two adjacent measures in one vector's amplitude will be proportional with the change in the other's amplitude. Such changes, if detected, lead to the elimination of both measures thus only the non influenced measures are retained.

Using this policy the number of measures can diminish thus eventually reducing the accuracy in determining the placing error but the use of thermal-drift influenced measures can increase the error in determining the holes' position and thus decreasing the accuracy. After this unwanted but necessary elimination all the other measures are averaged and then matched with an ideal "averaged" lattice.

This averaged ideal lattice is used further on for the statistical approach in order to find the mean error and the standard deviation both in Cartesian and Polar coordinates. Typical results of such calculations can be seen in figure 3 where the red stars indicate the averaged position while the blue arrows designate the (scaled) error vectors. The polar coordinates are useful in the case of a systematic error that can be averaged out in Cartesian coordinates.

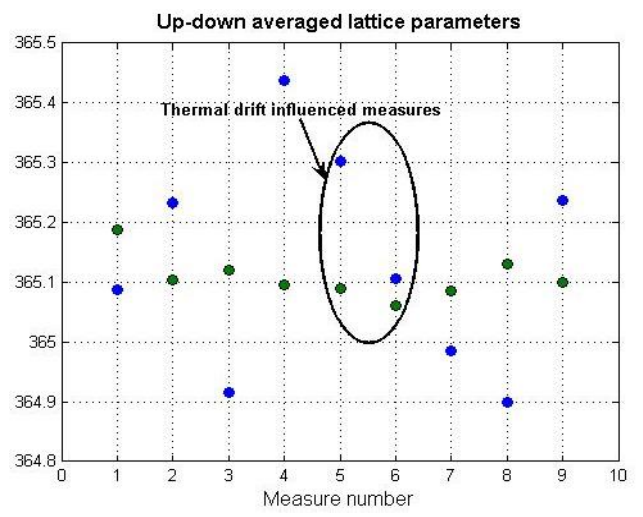

Figure 2. Matched lattice parameters graph. The vertical unit vector (blue) variance is proportional to the one of the 60 degrees oriented (green).

\section{RESULTS}

\section{A. Accuracy issues}

The standard AFM measure was performed in a 7X7 $\mu \mathrm{m}$ window enabling us to obtain an efficient field for statistical analysis. Presently, limitations due to the AFM software impose a maximum image dimension of 512 X 512 pixels. Thus, for our field of view, we obtain a pixel size of approximately $13.67 \mathrm{~nm}$. This pixel size may seem too large for nmdetermination the holes' positions, but for several reasons (see below) this pixel size is a good compromise for obtaining the desired accuracy.

First of all, since the accuracy of the AFM is below $1 \mathrm{~nm}$ the biggest relative error in determining the pixel's position is less than $7 \%$. A smaller pixel size would increase this error thus limiting the accuracy of the measure.

Next, the typical diameter of the measured holes is 180 $200 \mathrm{~nm}$ so a pixel dimension of $13.67 \mathrm{~nm}$ allows us to define the hole with around 180-200 pixels. Since the accuracy in defining the centre of mass is roughly proportional to the number of pixels the structure is defined by, this amount of pixels gives us the possibility of increasing the determination of the position's accuracy up to $\sim 0.1 \mathrm{~nm}$. In this approximate calculation of the centre of mass accuracy we didn't take into account the AFM intrinsic error but, since it is lower that $7 \%$, we can safely assume that the real accuracy for this determination is less than $0.11 \mathrm{~nm}$. A determination of the exact value is not the object of this paper. 


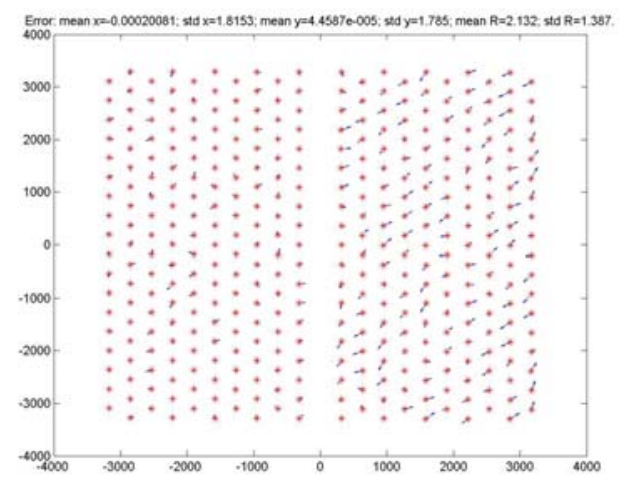

Figure 3. Typical results plot. The arrows indicate the error direction and amplitude (scaled to be visible)

Once determined, the centre of mass position accuracy for one measure, in order to determine the overall one, we shall divide this value to the number of measurements. To be noted that, from the statistical point of view, we do not make any errors since the measures were made considering the exact same structure and the only parameter that significantly varied between one measure and another is the time. This leads us to an overall accuracy of less than $0.01 \mathrm{~nm}$.

One could argue that the temperature can play a role in such measures and that it is never constant. Moreover, some measures were influenced by thermal drift so the errors can be substantial due to this factor. Still the algorithm we used for averaging out the measures takes into account the relative positions of the holes and not the absolute ones so the displacement of the measuring area with several $\mathrm{nm}$ does not influence the results. Since the expansion coefficient of $\mathrm{Si}$ at $300 \mathrm{~K}$ is $2.6^{*} 10^{-6} \mathrm{~K}^{-1}$ [6] we can safely conclude that $0.5 \mathrm{~K}$ difference (maximum allowed in the AFM environment) does not influence the measurement in a significant manner.

\section{B. Obtained results}

For most of the measured samples, the standard deviation of the holes' position from the perfect lattice position is in the range of $1.3-2 \mathrm{~nm}$, with a mean value lower than the measuring accuracy (for the $\mathrm{x}$ and $\mathrm{y}$ values).

From the polar coordinates, the mean value of the deviation in radius was about $2-4 \mathrm{~nm}$ function of the lithographic equipment used.

Due to these values and the theoretical aspects presented in literature [3] one can easily conclude that the nowadays technique allows the fabrication of photonic crystal structures with an accuracy lower that the theoretical limit for influencing the structure's functionality in terms of propagation losses. However, for PhC components utilizing e.g. defects for cavities this positioning error could be devastating for their functionality [7].

\section{CONCLUSIONS}

We presented a method for measuring the position of lowindex structures (air holes) inside a photonic crystal utilizing atomic force microscopy and how, by post-processing, one can reach a less than $0.01 \mathrm{~nm}$ accuracy.

Using this algorithm we determined that the standard deviation of the holes' position with respect to an ideal lattice is usually around $1.5-2 \mathrm{~nm}$ even if the samples are fabricated in different facilities and using different process steps. To be noted that this values hold true for state-of-the-art facilities available within Europe and are not to be used as reference for all fabricated structures. Each fabrication facility has its own process for obtaining such devices so the above determined values can vary.

Based on these values and considering the theoretical predicted limits [3] for having the structure's functionality influenced by the positioning errors we can safely assume that losses in such structures are mainly due to factors like surface roughness, sidewall roughness and sidewall angle while the ones introduced by the positioning errors can be neglected. However, since characteristics like Q-factors of PhC cavitybased components are very sensible to nm-error positioning [7] they can and are, in our opinion, limited by such errors.

\section{ACKNOWLEDGMENTS}

The authors wish to thank the ePIXnet NoE partners for allowing us to measure on structures fabricated in their centres and also to Danish Fundamental Metrology, in particular to Joergen Garnaes, for his support in measuring the photonic crystals and post-processing the measures.

\section{REFERENCES}

[1] A. Chutinan, S. Noda, "Effects of structural fluctuations on the photonic bandgap during fabrication of a photonic crystal", J. Opt. Soc. Am. B, Vol. 16(2), pp. 240-244, 1999

[2] H.-Y. Ryu, J.-K. Hwang, Y.-H. Lee, "Effect of size nonuniformities on the band gap of two-dimensional photonic crystals", Phys. Rev. B, vol. 59(8), pp. 54635469, 1999

[3] R. Ferrini et al., "Disorder-induced losses in planar photonic crystals", Optics Letters, vol. 31(10), pp. 14261428 , may 2006

[4] B.-S. Song, S. Noda, T. Asano, Y. Akahane, "Ultra-high$Q$ photonic doubleheterostructure nanocavity", Nature materials, vol. 4, pp. 207-210, march 2005

[5] M. Svalgaard, L.H. Frandsen, J. Garnæs, A. Kühle, "Local Lattice Imperfections of Planar Photonic Crystals", PECS-VII proceedings, april 2007

[6] http://www.ioffe.rssi.ru/SVA/NSM/Semicond/SiGe/therm al.html

[7] T. Asano, B.-S. Song, Y. Akahane, S. Noda, "Ultrahigh-Q Nanocavities in Two-Dimensional Photonic Crystal Slabs", IEEE Journal of Selected Topics in Quantum Electronics, vol. 12 (61), pp. 1123-1134, 2006 